\title{
Role of miRNA-21 expression in profibrillatory fibrotic remodeling and atrial fibrillation occurrence
}

\author{
Peng Cao\#, Wei Lian\#, Pradeep Hathurusinghe, Deepak Sharma, Cong Cong Han and Yanmin Xu* \\ Department of Cardiology, Tianjin Key Laboratory of Ionic-Molecular Function of Cardiovascular disease, Tianjin Institute of Cardiology, Second Hospital of \\ Tianjin Medical University, PR China \\ "Authors contributed equally
}

\begin{abstract}
Atrial fibrillation (AF) is the most frequent cardiac arrhythmia seen in clinical practice and is associated with pronounced morbidity and mortality. It was demonstrated that $\mathrm{AF}$ is associated with altered miRNA levels in atrial tissue and plasma. The regulatory function of specific miRNAs has been studied in the structural and electrical remodeling underlying AF, ischemic heart disease, cardiac hypertrophy, ion channel modification and extracellular matrix (ECM) formation. Furthermore, circulating miRNAs associated with AF could serve as potential biomarkers of the disease, whereas specific tissue miRNAs could become targets for therapy. Although treatment of AF has substantially advanced in recent years, numerous problems remain, and a fundamental breakthrough has not been made in the treatment of AF.
\end{abstract}

\section{Introduction}

Atrial fibrillation $(\mathrm{AF})$ is the most frequent cardiac arrhythmia seen in clinical practice [1] and is associated with pronounced morbidity and mortality. The most important risk factor for new-onset AF is ageing, and ageing of the population importantly contributes to the growing number of patients with AF. AF prevalence is expected to double by 2050 [2]. According to the American Heart Association, AF can be classified as first detected episode of AF, paroxysmal when AF is sustained for less than 7 days and terminates spontaneously (PAF), persistent when $\mathrm{AF}$ is sustained beyond 7 days (PersAF, can be long-standing when $\mathrm{AF}$ persists for more than 1 year), and permanent when cardioversion attempts have failed or have not been tried [3]. The diagnosis of AF is based on the evaluation of symptoms and intermittent rhythm monitoring; however, those approaches are often inconclusive or unsatisfactory; moreover, most patients are asymptomatic. Therefore, there is a demand for biomarkers of diagnostic and/or prognostic value in AF [4]. Although treatment of AF has substantially advanced in recent years, numerous problems remain, and a fundamental breakthrough has not been made in the treatment of AF. Recent studies have shown that microRNAs (miRNA) may be involved in the pathophysiology of $\mathrm{AF}$ [5]. MicroRNA is a class of non-coding ribonucleic acid molecules 22 nucleotides in length, which is usually bound to the 3 'UTR of miRNA, thereby inhibiting the translation process or promoting the degradation of miRNA [6]. miRNAs regulate approximately $30 \%$ of all protein-coding genes and have therefore been predicted to be involved in almost all cellular processes [7]. miRNA regulation in cardiovascular disease was first described in 2006, when specific miRNAs were found to be up- or down regulated in mouse models of cardiac hypertrophy and heart failure (HF). Then it was demonstrated that AF is associated with altered miRNA levels in atrial tissue and plasma [8]. The regulatory function of specific miRNAs has been studied in the structural and electrical remodeling underlying $\mathrm{AF}$, ischemic heart disease, cardiac hypertrophy, ion channel modification and ECM formation [9-17]. Furthermore, circulating miRNAs associated with AF could serve as potential biomarkers of the disease, whereas specific tissue miRNAs could become targets for therapy

\section{Overview of AF pathophysiology and contribution of remodeling}

There are 4 principal pathophysiological mechanisms contributing to AF: Electrical remodeling, Structural remodeling, Autonomic nervous system changes, and $\mathrm{Ca}^{2+}$ handling abnormalities. Each of these can result from cardiac disease conditions and promote the development of AF. Focal ectopic firing can maintain AF or trigger re-entrant AF through a re-entry maintaining substrate that has the appropriate conditions to allow re-entry to be induced and then sustained. Induction and maintenance of re-entry require a critical balance between refractory and conduction properties [18-21].

\section{Electrical remodeling}

Electrical remodeling is atrial electrophysiological properties that are governed by ion channels, pumps, and exchangers, any of which can be altered by atrial remodeling. Electrical remodeling can change the expression, function, or localization of connexins, altering cell-tocell electrical coupling and resulting in re-entry-promoting slow and/or heterogeneous conduction [22]. miRNAs involved in cardiac electrical remodeling are miR-1, miR-26, miR-208a, miR-328 and miR-499. Their target genes are encoding ion channels, connexins or proteins

${ }^{\star}$ Correspondence to: Yanmin XU, Department of Cardiology, Tianjin Key Laboratory of Ionic-Molecular Function of Cardiovascular disease, Tianjin Institute of Cardiology, Second Hospital of Tianjin Medical University, PR China, Tel: 0086-13702029695; Fax: 0086-22-88328119; E-mail: xuyanminphdmd@sina.com

Key words: miRNA-21, fibrotic remodeling, atrial fibrillation

Received: October 01, 2019; Accepted: October 14, 2019; Published: October 17,2019 
involved in calcium signaling and resulting in conduction slowing or shortening of the action potential duration, which are hallmarks of AF pathophysiology.

\section{Structural remodeling}

This is characterized by atrial enlargement and tissue fibrosis. Under some functional conditions, atrial dimension is a key determinant of the persistence of AF maintaining re-entry. Fibrosis promotes AF by interrupting fiber bundle continuity and causing local conduction disturbances $[23,24]$. Recent work suggests that structural remodeling might be $\mathrm{Ca}^{2+}$ dependent. miRNAs involved in cardiac structural remodeling are miR-21, miR-26, miR-29b, miR-30, miR-133 and miR-590. These miRNAs regulate genes encoding proteins that are involved in extracellular matrix turnover and pro- or antifibrotic signaling cascades leading to atrial fibrosis as the anatomical substrate for reentry.

\section{Autonomic nervous system}

Autonomic nervous system control regulates atrial bioelectricity and contributes to the initiation and maintenance of AF. Adrenergic activation increases $\mathrm{I}_{\mathrm{Ca}, \mathrm{L}}$, RyR2(ryanodine receptor type 2) open probability, and SR (sarcoplasmic Reticulum) $\mathrm{Ca}^{2+}$ load via phosphorylation by CaMKII ( $\mathrm{Ca}^{+} /$calmodulin-dependent protein kinase II;) and protein kinase A. The risk of DADs (delayed after depolarizations) is consequently enhanced, and Adrenergic activation may play a critical role in AF due to formation of ectopic activity in the context of various remodeling-induced paradigms [25,26]. Autonomic hyper innervation is a consequence of AF-related remodeling and contributes to the vulnerable AF substrate.

\section{$\mathrm{Ca}^{2+}$ handling abnormalities}

The most significant direct atrial profibrillatory consequence of $\mathrm{Ca}^{2+}$ handling abnormalities is the induction of DAD-related spontaneous atrial ectopic activity. Patients with long-standing persistent AF have an increased risk of arrhythmogenic DADs/ triggered activity [27]. There is recent evidence that rapid atrial rates may cause $\mathrm{Ca}^{2+}$ silencing. More work is clearly needed to clarify the role of abnormal $\mathrm{Ca}^{2+}$ handling in the generation of atrial ectopic activity and the initiation/maintenance of $\mathrm{AF}$ in specific patient populations. Atrial $\mathrm{Ca}^{2+}$ handling abnormalities associated with catecholaminergic polymorphic ventricular tachycardia (CPVT) reduce atrial conduction velocity (CV) by inhibiting Ina [28]. Computational modelling has also shown that sub threshold DADs can cause local conduction slowing and/or block by reducing Ina [29]. Finally, $\mathrm{Ca}^{2+}$ dependent signaling can contribute to atrial electrical and structural remodeling. Thus, in addition to focal ectopy, $\mathrm{Ca}^{2+}$ handling abnormalities can contribute to the re-entry-promoting substrate facilitating AF maintenance.

\section{MiR-21 and cardiac remodeling}

miRNA exerts a crucial role in the pathophysiological mechanism of arrhythmia [30]. Some miRNAs have been confirmed to be related to the occurrence and development of AF. (miR-21, miR-29, miR-133, miR-30, miR-590.32 miR-133 and miR-590). Among them miRNA-21 is strongly involved in myocardial fibrosis. Also, miRNA-21 knockout significantly inhibited the process of atrial fibrillation and the induction rate of AF after myocardial infarction [12,31-33]. Atrial fibrosis is a negative predictor for procedure outcome in patients undergoing ablation for persistent atrial fibrillation. However, noninvasive methods to determine the extent of left atrial fibrosis are limited. miR-21 is a major regulator of cardiac fibrosis. As a biomarker, it is detectable in blood. miRs can post transcriptionally silence protein expression either by binding to complementary target mRNAs and degrading these mRNAs, or by inhibiting mRNAs translating into proteins [34]. MIinduced heart failure leads to AF-promoting atrial remodeling in rats. Atrial miR-21 knockdown suppresses atrial fibrosis and AF promotion, implicating miR-21 as an important signaling molecule for the AF substrate and pointing to miR-21 as a potential target for molecular interventions designed to prevent AF. Interestingly, AF promotion was suppressed with reduced atrial fibrosis, despite persistent LA dilation. These findings are consistent with previous work in which AF promotion persisted along with continued atrial fibrosis, although LA dilation was reversed after hemodynamic recovery from HF [35]. These results suggest that atrial fibrosis is an essential factor in the HF-related AF substrate, providing support for upstream therapy that prevents or reverses atrial fibrosis in AF management. Although systemic antimiR-21 administration might protect against both HF-induced atrial structural remodeling and LV function impairment, side effects of systemic administration might be a clinical problem. For example, anti apoptotic effects of miR-21 are needed for ischemic preconditioning and prevent abdominal aortic aneurysm dilation. Local administration, though technically challenging, could overcome these barriers and provide direct, tissue-specific miR-21 inhibition [36-39]. Upregulation of miR-21, highly expressed in fibroblasts, has been associated with increased cardiac fibrosis, not limited to AF [12,32,36,40-42]. Several mechanisms have been proposed for the potential effect of miR-21 on fibrosis, but most attention has been paid to miR-21 repression of SPRY1 (Sprouty 1, RTK signaling antagonist). Sprouty-1 inhibits the extracellular signal-regulated kinases (ERK) signaling pathway, which promotes fibrosis. Upregulation of miR-21 was associated with downregulation of SPRY1 in patients with valvular AF and in rats with myocardial ischemia. In vivo inhibition with antagomir- 21 in mice or a 15-mer LNA-based anti-miR-21 in rats suppressed fibrosis and AF. However, in the heart failure field contradicting results about the role of miR-21 in fibrosis have been reported. Thum et al. [36] showed that inhibition of miR-21 by antagomir injection protected mice against cardiac fibrosis and attenuated cardiac dysfunction in response to thoracic aorta constriction (TAC). On the other hand, Patrick et al. [43] reported that neither genetic deletion of miR-21 nor inhibition with tiny LNA-based anti-miRs altered cardiac fibrosis in response to various stresses, like TAC and MI. Contradicting findings may results from different effectiveness of the anti-miR-21 and antago-mir-21 chemistries, used in the different studies [44]. Recent study was able to investigate the role of miR-21 and CADM1 in cardiac fibrosis and potential interaction between miR-21 and CADM1. More importantly, miR- 21 inhibitor repressed cardiac fibrosis progression, miR- 21 may be a better alternative to directly inhibit CADM1 expression in cardiac fibrosis. The mRNA and protein of STAT3 increased in activated fibroblast and fibrosis tissue. TGF- $\beta 1$ induced fibroblasts activation and proliferation through STAT3. However, CADM1 mRNA and protein expression was significantly decreased in cardiac fibrosis tissue and fibroblast. Studies demonstrated that CADM1 may inhibit STAT3 activity and control cellular proliferation. Therefore, CADM1/STAT3 pathway is important for cardiac fibrosis development. Emerging evidence suggests that miR-21 regulation of cardiac fibroblast activation and fibrosis. Also demonstrated that the expression of miR21 increased in cardiac fibroblast and fibrosis tissue. Also showed that silencing of miR-21 can inhibit a-SMA expression. Accumulating evidence implicates miR-21 in a variety of disorders and indicates that it is highly upregulated during cardiac fibrosis. The expression of miR21 is also increased in atrial tissues and plasma samples from patients with AF. Importantly, atrial miR-21 knockdown with antagomir-21 
suppresses atrial fibrosis and reduces AF promotion in rats after chronic myocardial infarction, making this molecule an effective target for AF treatment. Recent studies have demonstrated that STAT3 can regulate miR-21 expression moreover, miR-21 also modulates the STAT3 signaling pathway. In addition, given the importance of both STAT3 and miR-21 in the fibrotic processes linked to AF, we hypothesized that they might form a reciprocal positive loop that contributes to POAF by promoting atrial fibrosis [42].

\section{miR-21 is expressed in human atrial myocytes and is upregulated by CAF}

In some qPCR results demonstrated for the first time that miR-21 was expressed in enzymatically isolated atrial myocytes obtained from patients in SR. Some authors [45] suggested that miR-21 was expressed in mouse and rat cardiac myocytes, where it could play a role in cardiac hypertrophy and in the protective effect of ischemic preconditioning against ischemia-induced cardiac myocyte damage. On the contrary, other authors described that miR-21 was not expressed in mouse cardiac cells, and thus, its role in cardiac remodeling produced in a pressure overload-induced mouse model was attributed to its presence in fibroblasts that affects cardiac myocytes in a paracrine fashion. In human atria, miR-21 was found in whole atrial biopsies. However, it is generally accepted that the presence of fibroblasts and nonmyocyte cells in whole biopsies complicates gene expression analysis. From this article results, we suggest that, besides in fibroblasts, miR-21 is expressed in human atrial myocytes isolated from patients with CAF. Moreover, it decreases $\mathrm{I}_{\mathrm{Ca}, \mathrm{L}}$ density by downregulating $\mathrm{Ca}^{2+}$ channel subunits expression. These results suggested that miR-21 could participate in the CAF-induced $\mathrm{I}_{\mathrm{Ca}, \mathrm{L}}$ downregulation and in the APD shortening that maintains the arrhythmia.

\section{Future Perspective of miRNAs as therapeutics}

miRNAs hold a promise for the development of a new class of therapeutics as expression and function can be enhanced or repressed by the systemic or local delivery of synthetic miRNA mimics and inhibitors respectively. miRNA research in AF and their role in atrial cardiomyopathies lags behind research in the field of ventricular cardiomyopathies since the first human explorative studies in $\mathrm{AF}$ were performed in 2011 and most functional studies, besides a few exceptions, date from 2012 onwards. Meanwhile, progress has been made on miRNA-based therapies in pre-clinical trials for other cardiac diseases.

\section{Conclusion}

In this review, we present up-to-date evidence on the role of miRNAs in AF pathophysiology. Explorative studies have indicated tissue and plasma miRNAs to be differentially expressed in patients with $\mathrm{AF}$ and functional studies implicated miRNAs in several pathophysiological pathways. We found that miRNA-21 expression in human atrial tissue is potentially associated with atrial fibrosis and might affect AF occurrence, which would indicate its potential usefulness as a new class of biomarkers and promote the development of innovative therapies.

\section{References}

1. Kara K, Geisel MH, Möhlenkamp S, Lehmann N, Kälsch H, et al. (2015) B-type natriuretic peptide for incident atrial fibrillation-The Heinz Nixdorf Recall Study. $J$ Cardiol 65: 453-458. [Crossref]

2. Ball J, Carrington MJ, McMurray JJ, Stewart S (2013) Atrial fibrillation: profile and burden of an evolving epidemic in the 21st century. Int J Cardiol 167: 1807-1824. [Crossref]
3. Zipes DP, Camm AJ, Borggrefe M, Buxton AE, Chaitman B, et al. (2006) ACC/AHA ESC 2006 guidelines for management of patients with ventricular arrhythmias and the prevention of sudden cardiac death--executive summary: A report of the American College of Cardiology/American Heart Association Task Force and the European Society of Cardiology Committee for Practice Guidelines (Writing Committee to Develop Guidelines for Management of Patients with Ventricular Arrhythmias and the Prevention of Sudden Cardiac Death) Developed in collaboration with the European Heart Rhythm Association and the Heart Rhythm Society. Eur Heart J 27: 2099-2140. [Crossref]

4. Carlos A, Pimenta E, Drager LF (2016) "Diretrizes Brasileiras de Hipertensão VI" Capitulo 7 Hipertensão Arterial Sistêmica Secundária 2016: 106: 44-53.

5. Zhang K, Zhao L, Ma Z, Wang W, Li X, et al. (2018) Doxycycline Attenuates Atrial Remodeling by Interfering with MicroRNA-21 and Downstream Phosphatase and Tensin Homolog (PTEN)/Phosphoinositide 3-Kinase (PI3K) Signaling Pathway. Med Sci Monit 24: 5580-5587. [Crossref]

6. Santulli G, Iaccarino G, De Luca N, Trimarco B, Condorelli G (2014) Atrial fibrillation and microRNAs. Front Physiol 5: 15. [Crossref]

7. Filipowicz W, Bhattacharyya SN, Sonenberg N (2008) Mechanisms of posttranscriptional regulation by microRNAs: are the answers in sight? Nat Rev Genet 2008: 102-114. [Crossref]

8. Luo X, Yang B, Nattel S (2015) MicroRNAs and atrial fibrillation: mechanisms and translational potential. Nat Rev Cardiol 12: 80-90. [Crossref]

9. van Rooij E, Sutherland LB, Thatcher JE, DiMaio JM, Naseem RH, et al. (2008) Dysregulation of microRNAs after myocardial infarction reveals a role of miR-29 in cardiac fibrosis. Proc Natl Acad Sci U S A 105: 13027-13032. [Crossref]

10. Girmatsion Z, Biliczki P, Bonauer A, Wimmer-Greinecker G, Scherer M, et al (2009) Changes in microRNA-1 expression and IK1 up-regulation in human atrial fibrillation. Heart Rhythm 6: 1802-1809. [Crossref]

11. Qi XY, Huang H, Ordog B, Luo X, Naud P, et al. (2015) Fibroblast inward-rectifier potassium current upregulation in profibrillatory atrial remodeling. Circ Res 116: 836845. [Crossref]

12. Adam O, Löhfelm B, Thum T, Gupta SK, Puhl SL, et al. (2012) Role of miR-21 in the pathogenesis of atrial fibrosis. Basic Res Cardiol 107: 278. [Crossref]

13. Cardin S, Guasch E, Luo X, Naud P, Le Quang K, et al. (2012) Role forMicroRNA-21 in atrial profibrillatory fibrotic remodeling associated with experimental postinfarction heart failure. Circ Arrhythm Electrophysiol 5: 1027-1035. [Crossref]

14. Duisters RF, Tijsen AJ, Schroen B, Leenders JJ, Lentink V, et al. (2009) miR-133 and miR-30 regulate connective tissue growth factor: implications for a role of microRNAs in myocardial matrix remodeling. Circ Res 104: 170-178, 6p following 178. [Crossref]

15. Chen X, Ba Y, Ma L, Cai X, Yin Y, et al. (2008) Characterization of microRNAs in serum: a novel class of biomarkers for diagnosis of cancer and other diseases. Cell Res 18: 997-1006. [Crossref]

16. van Rooij E, Olson EN (2012) MicroRNA therapeutics for cardiovascular disease: opportunities and obstacles. Nat Rev Drug Discov 11: 860-872. [Crossref]

17. Weckbach LT, Grabmaier U, Clauss S, Wakili R (2016) MicroRNAs as a diagnostic tool for heart failure and atrial fibrillation. Curr Opin Pharmacol 27: 24-30. [Crossref]

18. Heeringa J, van der Kuip DA, Hofman A, Kors JA, van Herpen G, et al. (2006) Prevalence, incidence and lifetime risk of atrial fibrillation: the Rotterdam study. Eur Heart J 27: 219-226.

19. Wakili R, Voigt N, Kääb S, Dobrev D, Nattel S (2011) Recent advances in the molecular pathophysiology of atrial fibrillation. J Clin Invest 121: 2955-2968. [Crossref]

20. Nattel S, Burstein B, Dobrev D (2008) Atrial remodeling and atrial fibrillation: mechanisms and implications. Circ Arrhythm Electrophysiol 1: 62-73. [Crossref]

21. Nattel S, Dobrev D (2012) The multidimensional role of calcium in atrial fibrillation pathophysiology: mechanistic insights and therapeutic opportunities. Eur Heart J 33: 1870-1877. [Crossref]

22. Kato T, Iwasaki YK, Nattel S (2012) Connexins and atrial fibrillation: filling in the gaps. Circulation 125: 203-206. [Crossref]

23. Zou R, Kneller J, Leon LJ, Nattel S (2005) Substrate size as a determinant of fibrillatory activity maintenance in a mathematical model of canineatrium. Am J Physiol Heart Circ Physiol 289: H1002-1012. [Crossref]

24. Burstein B, Comtois P, Michael G, Nishida K, Villeneuve L, et al. (2009) Changes in connexin expression and the atrial fibrillation substrate in congestive heart failure. Circ Res 105: 1213-1222. [Crossref] 
25. Nishida K, Qi XY, Wakili R, Comtois P, Chartier D, et al. (2011) Mechanisms of atrial tachyarrhythmias associated with coronary artery occlusion in a chronic canine model. Circulation 123: 137-146. [Crossref]

26. Choi EK, Shen MJ, Han S, Kim D, Hwang S, et al. (2010) Intrinsic cardiac nerve activity and paroxysmal atrial tachyarrhythmia in ambulatory dogs. Circulation 121 2615-2623. [Crossref]

27. Voigt N, Li N, Wang Q, Wang W, Trafford AW, et al. (2012) Enhanced sarcoplasmic reticulum $\mathrm{Ca} 2+$ leak and increased $\mathrm{Na}+-\mathrm{Ca} 2+$ exchanger function underlie delayed afterdepolarizations in patients with chronic atrial fibrillation. Circulation 125: 20592070. [Crossref]

28. King JH, Wickramarachchi C, Kua K, Du Y, Jeevaratnam K, et al. (2013) Loss of Nav1.5 expression and function in murine atria containing the RyR2-P2328S gain-offunction mutation. Cardiovasc Res 99: 751-759. [Crossref]

29. Liu MB, de Lange E, Garfinkel A, Weiss JN, Qu Z (2015) Delayed afterdepolarizations generate both triggers and a vulnerable substrate promoting reentry in cardiac tissue. Heart Rhythm 12: 2115-2124. [Crossref]

30. Wojciechowska A, Braniewska A, Kozar-Kami̊̊,ska K (2017) MicroRNA in cardiovascular biology and disease. Adv Clin Exp Med 26: 865-874. [Crossref]

31. van den Berg NWE, Kawasaki M, Berger WR, Neefs J, Meulendijks E, et al. (2017) MicroRNAs in atrial fibrillation: From expression signatures to functional implications. Cardiovasc Drugs Ther 31:345-365. [Crossref]

32. Cardin S, Guasch E, Luo X, Naud P, Le Quang K, et al. (2012) Role for MicroRNA-21 in atrial profibrillatory fibrotic remodeling associated with experimental postinfarction heart failure. Circ Arrhythm Electrophysiol 5: 1027-1035. [Crossref]

33. Bhattacharyya P, Nag S, Bardhan S, Acharya D, Paul R, et al. (2009) The role of longterm doxycycline in patients of idiopathic pulmonaryfibrosis: The results of an open prospective trial. Lung India 26: 81-85. [Crossref]

34. Dong X, Liu S, Zhang L, Yu S, Huo L, et al. (2015) Downregulation of miR-21 is involved in direct actions of ursolic acid on the heart: implications for cardiac fibrosis and hypertrophy. Cardiovasc Ther 33: 161-167. [Crossref]

35. Shinagawa K, Shi YF, Tardif JC, Leung TK, Nattel S (2002) Dynamic nature of atrial fibrillation substrate during development and reversal of heart failure in dogs. Circulation 105: 2672-2678. [Crossref]
36. Thum T, Gross C, Fiedler J, Fischer T, Kissler S, et al. (2008) MicroRNA-21 contributes to myocardial disease by stimulating MAP kinase signalling in fibroblasts. Nature 456: 980-984. [Crossref]

37. Savelieva I, Kakouros N, Kourliouros A, Camm AJ (2011) Upstream therapies for management of atrial fibrillation: review of clinical evidence and implications for European Society of Cardiology guidelines. Europace 13: 308-328. [Crossref]

38. Yin C, Salloum FN, Kukreja RC (2009) A novel role of microRNA in late preconditioning: upregulation of endothelial nitric oxide synthase and heat shock protein 70. Circ Res 104: 572-575. [Crossref]

39. Maegdefessel L, Azuma J, Toh R, Deng A, Merk DR, et al. (2012) MicroRNA-21 blocks abdominal aortic aneurysm development and nicotine-augmented expansion. Sci Transl Med 4: 122ra22. [Crossref]

40. Adam O, Zimmer C, Hanke N, Hartmann RW, Klemmer B, et al. (2015) Inhibition of aldosterone synthase (CYP11B2) by torasemide prevents atrial fibrosis and atrial fibrillation in mice. J Mol Cell Cardiol 85: 140-150. [Crossref]

41. He X, Zhang K, Gao X, Li L, Tan H, et al. (2016) Rapid atrial pacing induces myocardial fibrosis by down-regulating Smad7 via microRNA-21 in rabbit. Heart Vessels: 1696-1708. [Crossref]

42. Huang Z, Chen XJ, Qian C, Dong Q, Ding D, et al. (2016) Signal transducer and activator of transcription 3/MicroRNA-21 feedback loop contributes to atrial fibrillation by promoting atrial fibrosis in a rat sterile pericarditis model. Circ Arrhythm Electrophysiol 9: 110-122. [Crossref]

43. Patrick DM, Montgomery RL, Qi X, Obad S, Kauppinen S, et al. (2010) Stressdependent cardiac remodeling occurs in the absence of microRNA-21 in mice. J Clin Invest 120: 3912-3916. [Crossref]

44. Tijsen AJ, Pinto YM, Creemers EE (2012) Non-cardiomyocyte microRNAs in heart failure. Cardiovasc Res 93: 573-582. [Crossref]

45. Barana A, Matamoros M, Dolz-Gaitón P, Pérez-Hernández M, Amorós I, et al. (2014) Chronic Atrial Fibrillation Increases MicroRNA-21 in Human Atrial Myocytes Decreasing L-Type Calcium Current. Circ Arrhythm Electrophysiol 7: 861-868. [Crossref]

Copyright: (C2019 Cao P. This is an open-access article distributed under the terms of the Creative Commons Attribution License, which permits unrestricted use, distribution, and reproduction in any medium, provided the original author and source are credited. 